\title{
Srikumar P. Chellappan: Chromatin protocols
}

\author{
Methods in molecular biology 523, 2nd edition. Springer, 2009, HB, 416 pp, \\ EUR 74,95. ISBN: 978-1-58829-873-7
}

\author{
Ray Waters
}

Published online: 21 August 2009

(C) Springer-Verlag 2009

This book contains a collection of detailed and useful procedures concerning chromatin analysis. It includes methods for the isolation and assembly of nucleosomes, the analysis of basic chromatin structure, the covalent modification of histones and the analysis of chromatin function.

It then goes on to consider techniques to study how DNA repair functions in the context of chromatin. Here, there is much focus on the analysis of double strand break repair and the role of histone $\mathrm{H} 2 \mathrm{AX}$ phosphorylation. There is some mention of nucleotide excision repair but with a tight focus on the transcription-coupled aspects of this pathway. Surprisingly, there is neither the mention of global genome nucleotide excision repair (where a number of groups have made inroads into how GG-NER operates on repressed genes in chromatin), nor of photoreactivation repair and where Thoma has made some significant contributions.

This section is followed by one that considers the roles of histone and non-histone DNA-bound proteins in DNA replication. The book then goes on to discuss the roles of telomeres in genome integrity. Here, methods to describe telomere integrity and length are described. With respect to the estimation of telomere length, there is a startling omission in that the elegant approach developed by Baird has been ignored. That powerful method can examine telomere length at nucleotide resolution, and it has resulted in several publications concerning telomere maintenance in eminent journals. That approach is far more powerful than the one described.

It was good to see extensive discussion of methods employing ChIP on chip with tiling arrays to examine chromatin modifications; such approaches have made significant inroads into our understanding of chromatin structure and function in entire genomes. Here, it was a pity that there was no mention of the Chip-seq approaches that have emerged in the last few years, and which can provide an even more detailed insight into how entire genomes are organised and function.

In summary, this is a very useful text, with lots of strengths and just a few weaknesses.

R. Waters $(\bowtie)$

Department of Pathology, Cardiff University,

Tenovus Building, Heath Park, Cardiff, UK

e-mail: WATERSR1@ cardiff.ac.uk 